\title{
Qualidade de vida das pacientes mastectomizadas atendidas pelo serviço de fisioterapia do Hospital Universitário da Universidade Estadual de Londrina
}

\section{Quality of life of patient who undergoing mastectomies assisted by the service of physiotherapy of University Hospital of Londrina State University}

\author{
Eliane Cristina Hilberath Moreira ${ }^{1}$; Clárcia Aparecida Rodrigues Manaia ${ }^{2}$
}

\begin{abstract}
Resumo
Na paciente submetida ao tratamento cirúrgico do câncer de mama, o estigma da doença, a mutilação, a estética, a limitação nas atividades da vida diária após a cirurgia, a rotina de exames, o tratamento e das seqüelas de curto e médio prazo não são os únicos problemas apresentados. A ausência ou alteração da mama traz efeitos físicos, psicossociais, sexuais e emocionais que irão afetar diretamente a qualidade de vida dessas pacientes. Este estudo teve por objetivo verificar a qualidade de vida em mulheres mastectomizadas atendidas pelo serviço de fisioterapia da Universidade Estadual de Londrina. Utilizouse o questionário de qualidade de vida específico para pacientes mastectomizadas, desenvolvido pelo ambulatório de fisioterapia em ginecologia e obstetrícia da Universidade Estadual de Londrina em 2003, aplicado em dois momentos. Notou-se uma diferença estatisticamente significativa na comparação entre a qualidade de vida pré e pós-tratamento fisioterápico $(\mathrm{p}<0,05)$. Observou-se que houve melhora da qualidade de vida das pacientes atendidas pela clínica de fisioterapia indicando que o tratamento fisioterápico, conservador, de baixo custo possui benefícios na reabilitação da paciente mastectomizada, sendo necessário mais estudos com uma população maior para afirmação dos resultados.
\end{abstract}

Palavras-chave: Fisioterapia. Qualidade de vida. Mastectomia.

\begin{abstract}
As for the patient submitted to breast cancer surgical treatment, the stigma of the disease, the mutilation, the aesthetics, the limitation in the daily life activities after the surgery, the exams routine, the treatment and the short and medium term sequelae are not the only problems to be solved. The breast removal or the aesthetic alteration brings physical, psychological, sexual and emotional problems that will affect their quality of life. The purpose of this study was to verify the quality of life in women who are submitted to mastectomy, assisted by the physiotherapists of the State University of Londrina, using the Quality of Life Specific Questionnaire. This questionnaire was developed by the gynecology and obstetrics physiotherapy clinics of the State University of Londrina in 2003, and was applied before and after the physiotherapy treatment. A significant statistical difference was observed in the quality of life before and after the treatment $(p<0,05)$. It was observed an improvement of life quality of patients assisted by the physiotherapy clinic pointing out that the low-cost conservative physiotherapy treatment benefits the patient's rehabilitation. It also shows that a study with a larger population is necessary in order to confirm the results.
\end{abstract}

Key words: Physiotherapy. Life quality. Mastectomy.

${ }^{1}$ Professora Adjunta Doutora do Departamento de Fisioterapia da Universidade Estadual de Londrina/UEL. E-mail: liamoreira@uel.br

${ }^{2}$ Aluna do $4^{\circ}$ ano do curso de Fisioterapia da Universidade Estadual de Londrina - bolsista PIBIC/CNPQ.

Semina: Ciências Biológicas e da Saúde, Londrina, v. 26, n. 1, p. 21 -30, jan./jun. 2005 


\section{Introdução}

Mulheres com câncer de mama acumulam experiências de ajustamento psicológico durante o ciclo de tratamento. Intervenções que possam ajudar a reduzir o impacto negativo do tratamento na qualidade de vida devem ser designadas e integradas nas rotinas de prática clínica (NAGEL et al., 2001).

O avanço da conscientização da prevenção, da detecção precoce e da modernização no tratamento, trouxe como resultado o declínio da mortalidade por câncer de mama e levou um número maior de mulheres, que formam o grupo das sobreviventes, a valorizarem os fatores psicossociais e a qualidade de vida (SHIZOMUMA, 1999; SALES et al., 2001; HELGESON; SNYDER; SELTMAN, 2004). Há grande interesse em avaliar a eficácia de diversas modalidades de tratamentos para o câncer de mama, considerando além da taxa de mortalidade, o aumento na qualidade de vida dessas pacientes (NAGEL et al., 2001).

Há o consenso clínico de que a paciente deve ser tratada como um todo, globalmente. Para isso, são utilizados tanto parâmetros fisiológicos, quanto psicológicos (BRUNETTO; ZAMPIERI, 1997). Na paciente submetida à mastectomia, o estigma do câncer, a mutilação, a estética, a limitação nas atividades da vida diária após a cirurgia, a rotina de exames, e as complicações não são os únicos problemas apresentados. A ausência da mama traz, além dos efeitos físicos, conseqüências sociais e emocionais que irão afetar diretamente a qualidade de vida dessas mulheres. As conseqüências destas disfunções superam o marco individual e estendemse aos familiares, amigos e relações profissionais. Isso implica em uma sobrecarga emocional para a paciente (WHELAN et al., 2000; KRISHNAN et al., 2001; MOREIRA et al., 2002).

O tratamento fisioterapêutico para as pacientes submetidas à cirurgia para tratamento do câncer de mama deve começar no pré-operatório, estendendose ao pós-operatório. No Hospital Universitário, após a alta, a paciente é encaminhada para atendimento ambulatorial individual e, em seguida, em grupos. O fisioterapeuta tem como objetivo o alívio dos sintomas e o bem-estar da paciente, minimizando as disfunções físicas, prevenindo e/ou restabelecendo a perda da função, da força muscular e da amplitude de movimento da cintura escapular, desvios posturais, drenagem linfática. Esse trabalho visa permitir o retorno o mais breve possível às suas atividades diárias e profissionais, melhorando, dessa forma, a qualidade de vida.

Para avaliar uma paciente mastectomizada como um todo, devem ser examinados vários aspectos: o grau dos sintomas, o estado emocional, o estado do humor e a adaptação à nova aparência física. Esses aspectos podem ser medidos por meio de instrumentos quantitativos, como os questionários de qualidade de vida utilizados para este fim. Os instrumentos destinados à mensuração da qualidade de vida podem ser divididos em duas categorias: gerais e específicos. Ambos possuem a mesma função, porém suas características particulares justificam a eleição dos questionários específicos como os mais indicados para o uso clínico. Os instrumentos gerais utilizam-se de perguntas amplas a respeito de como a doença afeta a atividade diária do paciente, e permitem obter respostas geralmente representadas por uma escala compacta que varia do "pouco" ou "nada" ao "muito". Eles possuem caráter abrangente, podendo ser utilizados para doenças crônicas em geral, e são igualmente curtos e de simples administração, porém, em sua maioria, não possuem o requisito sensibilidade. Os instrumentos específicos, por sua vez, são relacionados a determinados distúrbios na vida diária e bem-estar de pacientes acometidos por uma patologia crônica em especial. Envolvem perguntas mais direcionadas à sintomatologia do entrevistado e requer maior complexidade na administração quando comparados aos instrumentos gerais. Grande parte desses instrumentos são comprovadamente sensíveis, reprodutíveis e válidos (GUYATT; FUNY; PATRICK, 1993; MCSWEENNY; CREER, 1995). 
A aplicação das medidas gerais e específicas de qualidade de vida tem sido citada, por vários estudiosos, com diferentes objetivos. Jones et al. (1992) propõem a utilização das medidas gerais em pesquisas que visam a avaliar o custo-efetividade, os efeitos colaterais e grau de invasividade de tratamentos aplicados a uma mesma patologia.

Com relação às medidas específicas, sua aplicação tem sido sugerida por Guyatt et al. (1987) e Guyatt, Funy e Patrick (1993) com objetivos clínicos. Segundo o autor, a delimitação das áreas de comprometimento da qualidade de vida do paciente é importante, tanto na mensuração do benefício do tratamento executado, quanto na elaboração de um tratamento ideal.

As principais razões para que o fisioterapeuta se preocupe em verificar a qualidade de vida de suas pacientes são: justificar diferentes formas de terapêuticas de tratamento, identificar as seqüelas de doenças, provar a eficácia de determinados métodos, e identificar quanto uma determinada alteração ou doença interferem na vida do indivíduo.

O objetivo deste estudo é verificar se a intervenção fisioterapêutica teve interferências na qualidade de vida de mulheres mastectomizadas, o trabalho foi realizado por meio da aplicação de um questionário de qualidade de vida, específico para pacientes submetidas ao tratamento cirúrgico de câncer de mama. Esse questionário foi desenvolvido na Universidade Estadual de Londrina - ambulatório de fisioterapia em ginecologia e obstetrícia (MOREIRA et al., 2002; MOREIRA et al., 2003).

\section{Casuística e Métodos}

O presente estudo foi realizado com a participação de 14 mulheres, com idade entre 37 a 69 anos, submetidas a mastectomia atendidas no ambulatório de fisioterapia em ginecologia e obstetrícia do Hospital Universitário de Londrina, no período de 2002 a 2004. Ele destina-se verificar o impacto do tratamento fisioterápico na qualidade de vida das mulheres mastectomizadas.
Utilizou-se o questionário de qualidade de vida específico para pacientes mastectomizadas, desenvolvido pela equipe do ambulatório de fisioterapia em ginecologia e obstetrícia do Hospital Universitário/UEL. E em outro estudo (MOREIRA et al., 2003), esse instrumento foi correlacionado com o questionário de qualidade de vida geral validado para o português SF-36, e isso permitiu verificar que o primeiro possui maior correlação significativa e especificidade pois são atribuídos valores entre 0 (zero) para pior qualidade de vida e 10 (dez) para ótima qualidade de vida. O questionário específico foi aplicado em duas etapas: a primeira mede a limitação física da paciente e nela o entrevistador apresenta à paciente, várias fichas com atividades da vida diária, que são mostradas progressivamente, para que esta identifique quais as atividades difíceis ou impossíveis de serem realizadas, devido às seqüelas cirúrgicas. Após a seleção das atividades, a paciente escolhe as cinco atividades que realiza com maior dificuldade. Então, uma escala visual analógica (EVA) de sete pontos é utilizada para classificar a intensidade da limitação sentida pela paciente durante a realização de cada atividade com a seguinte classificação: $1=$ não realizou a atividade sozinha; 2 = extremamente difícil, porém sozinha; $3=$ muito difícil; $4=$ dificuldade moderada; $5=$ pouco difícil; $6=$ pouquíssima dificuldade; $7=$ sem nenhuma dificuldade. Os resultados eram anotados em uma ficha para posterior cálculo da nota. A segunda etapa do questionário contém 25 questões avaliando a aparência física, estado emocional, sexualidade e sintomatologia. Cada uma das questões foi respondida por meio de outra escala, também de sete pontos; $1=$ sempre; $2=$ quase o tempo todo; $3=$ a maior parte do tempo; $4=$ metade do tempo; $5=$ poucas vezes; $6=$ raramente; $7=$ nunca. Para cada uma dessas áreas, foi calculada a média de cada uma delas e então, determinado o valor médio da qualidade de vida da paciente (Anexo 1).

As entrevistas ocorreram em salas fechadas do ambulatório por um entrevistador devidamente treinado para a sua aplicação antes e após o 
tratamento fisioterápico, com tempo de aplicação de 30 minutos.

Após a aplicação dos questionários, utilizou-se o teste $\mathrm{T}$ pareado do programa SPSS 11.5 for Windows com significância de $5 \%(\mathrm{p}<0,05)$ para a comparação dos questionários aplicados antes e após o tratamento fisioterápico. Este estudo foi submetido e aprovado pelo comitê de ética da Universidade Estadual de Londrina, e todas as pacientes preencheram o termo de consentimento livre e esclarecido.

\section{Resultados e Discussão}

Segundo a Organização Mundial de Saúde (OMS), a qualidade de vida é definida como "a percepção do indivíduo de sua posição na vida, no contexto da cultura e sistemas de valores, nos quais ele vive em relação aos seus objetivos, expectativas, padrões e preocupações". Ela também é descrita como uma sensação de bem estar na realização das atividades físicas, intelectuais e psíquicas, dentro da realidade particular de cada indivíduo (SALES et al., 2001).

Em 2001, Nagel et al. observaram que sobreviventes do câncer de mama alcançam recuperação máxima fisicamente e psicologicamente no prazo de um ano após a cirurgia. Contudo, há evidências de declínio nos dois anos seguintes, indicando a necessidade de análise das técnicas, ajustamentos de tratamentos e estratégias de reabilitação.

Burnham, Timothy e Wilcox (2002) observaram que, em pacientes com câncer, o exercício pode melhorar a capacidade aeróbica, a capacidade de percorrer distâncias, a força muscular e a alteração da imagem corporal, reduzir náuseas, fadiga e melhorar a qualidade de vida.

Este estudo está de acordo com dados obtidos na literatura, pois o período médio de tratamento fisioterápico aplicado no ambulatório individual do $\mathrm{HU}$ foi de, aproximadamente, 8 meses $(7,9 \pm 8,7)$. Há, porém, discordâncias acerca do declínio do tratamento, pois até o presente momento não houve retorno das mulheres após o tratamento aplicado.

$\mathrm{Na}$ análise estatística para os parâmetros limitação física, sexualidade e qualidade de vida total, houve diferença estatisticamente significante entre a avaliação inicial e pós-tratamento $(p<0,05)$, (Tabela 1), indicando que o tratamento fisioterápico promove a melhora ou redução da disfunção da amplitude de movimento e da força muscular da cintura escapular, a reeducação /correção postural, a imagem corporal e a auto-estima dessas mulheres.

Tabela 1. Teste T pareado para a comparação entre a qualidade de vida de mulheres mastectomizadas antes e após o tratamento fisioterápico.

\begin{tabular}{l|c|c|c|c|c}
\hline \multicolumn{1}{c|}{ ASPECTOS AVALIADOS } & MÉDIA & DESVIO PADRÃO & ERRO PADRÃO & $\mathrm{T}$ & $\begin{array}{c}\text { SIGNIFICÂNCIA } \\
\text { (BI-CAUDAL) }\end{array}$ \\
\hline LIMITAÇÃO FÍSICA & $-2,8593$ & 3,44598 &, 92098 & $-3,105$ &, 008 \\
\hline SINTOMATOLOGIA & $-2,6086$ & 2,19329 &, 58618 & $-4,450$ &, 001 \\
\hline EMOCIONAL & $-1,1686$ & 2,19028 &, 58538 & $-1,996$ &, 067 \\
\hline ASPÉCTO FÍSICO & $-1,0100$ & 2,72630 &, 72863 & $-1,386$ &, 189 \\
\hline SEXUALIDADE & $-2,2743$ & 2,82189 &, 75418 & $-3,016$ &, 010 \\
\hline QUALIDADE DE VIDA TOTAL & $-1,8857$ & 1,43288 &, 38295 & $-4,924$ &, 000 \\
\hline
\end{tabular}

A dor crônica, o linfedema, a neuropatia pósirradiação e outros sintomas são encontrados em $75 \%$ das mulheres após tratamento do câncer de mama (GROUTTRUP et al., 2000; VELANOVICH; SZYMANSKI, 1999).
Segundo Hack et al. (1999), 72\% das mulheres apresentam dor no ombro e braço, fraqueza muscular ou torpor na semana depois da radioterapia, sendo a qualidade de vida afetada por todos esses fatores. 
$\mathrm{O}$ presente estudo indica que o tratamento fisioterápico oferece às pacientes melhora no desconforto causado após o tratamento, pois quando analisado o parâmetro sintomatologia houve diferenças estatisticamente significativas antes e após a intervenção fisioterapêutica.

O estudo realizado em 2001 por Nagel et al. conclui que mulheres com câncer de mama podem apresentar ajustamentos psicológicos em vários pontos do ciclo de tratamento.

As pacientes apresentam problemas psicológicos de ajustamento relacionados à aceitação dos limites impostos pela sua enfermidade, devendo, assim, procurar compreender suas atitudes, a fim de ajudálas a assumir os aspectos positivos de sua personalidade (CAMARGO; MARX, 2000; MATSUOKA et al., 2002).

$\mathrm{Na}$ análise dos itens emocional e aspecto físico, não houve diferença estatisticamente significante (Tabela 1), e isso indica a necessidade da abordagem interdisciplinar, principalmente com psicólogos e outros profissionais. Com isso, será possível obter o ajustamento psicológico, e aceitação da enfermidade pelos familiares.

Todo tipo de integração da paciente com o meio social é realizado por meio de participação em grupo ou por terapias que façam redescobrirem seu corpo e sua capacidade de agir com ele. Com isso, oferecese à paciente melhora na auto-estima e, assim, melhorando sua qualidade de vida.

Mulheres submetidas à quimioterapia apresentam redução da satisfação com a vida sexual. Ao comparar mulheres que realizaram quimioterapia após a cirurgia com mulheres submetidas apenas à cirurgia, aquelas referem a redução da satisfação sexual duas vezes maior que as mulheres não submetidas ao tratamento quimioterápico (NAGEL et al., 2001).

\section{Conclusão}

Este estudo indica que a atuação fisioterapêutica no tratamento da paciente submetida a mastectomia promove melhora da qualidade de vida total, e em alguns parâmetros avaliados como limitação física e sexualidade, sendo necessários maiores estudos com maior número de indivíduos e estudo comparativo entre a qualidade de vida de mulheres mastectomizadas tratadas e não tratadas pela fisioterapia, além de estudos com abordagem interdisciplinar para a confirmação dos resultados obtidos.

\section{Referências}

BRUNETTO, A. F.; ZAMPIERI, C. O questionário de qualidade de vida na avaliação do DPOC, uma ferramenta útil para o fisioterapeuta. Fisioterapia em movimento, Curitiba, v.10, n.1, p.23-30, 1997.

BURNHAM, T. R.; TIMOTHY, R.; WILCOX, A. Effects of exercise on physiological and psychological variables in cancer survivors. Medicine \& Science in Sports \& Exercise, Baltimore, v.34, n.12, p.1863-1867, Dec. 2002.

CAMARGO, M. C.; MARX, G. Dor e outras patologias associadas ao câncer de mama. In: . Reabilitação física no câncer de mama. São Paulo: Roca, 2000. p.139146.

GROUTTRUP, H.; ANDERSEN, J.; ARENDT-NILSEN, L.; JENSEN, T. S. Psychophysical examination in patients with post-mastectomy pain. Pain, Amsterdam, v.87, n.3, p.275284, sep. 2000.

GUYATT, G. H.; BERMAN, L. B.; TOWSEND, M.; PUGSLEY, S. O; CHAMBERS, L. W. A measure of quality of life for clinical trials in chronic lung disease. Thorax, London, v.42, p.773-778, 1987a.

GUYATT, G. H.; BERMAN, L. B.; TOWSEND, M.; PUGSLEY, S. O.; CHAMBERS, L. W. Quality of life in pacients with chronic airflow limitation. British Journal of Disease in Chest, London, v.81, p.45-54, 1987b.

GUYATT, G. H.; FUNY, D. H.; PATRICK, D. L. Measuring Health-related Quality of Life. Annals of Internal Medicine, Philadelphia, v.118, n.8, p.622-629, 1993.

HACK, T. F.; COHEN, L.; KATZ, J.; ROBSON, L. S.; GOSS, P. Physical and psychological morbidity after axillary lymph node dissection for breast cancer. Journal of Clinical Oncology, Baltimore, v.17, n.1, p.143-149, Jan. 1999. 
HELGESON, V. S.; SNYDER, P.; SELTMAN, H. Pychological and physical adjustment to breast cancer over 4 year: Identfying distintict trajetrories of change. American Psycnological Association, New York, v.23, n.13,p.3-15, 2004.

JONES, P. W.; QUIRK, H.; BAVERYSTOCK, C. M.; LITTLEJOHNS, P. A Self-Complete measure of Health Status for Chronic Airflow Limitation. American Review of Respiratory Disease, New York, v.145, p.1321-1327, 1992.

KRISHNAN, L.; STANTON, A. L.; COLLINS, C. A.; LISTON, V. E.; JEWELL, W. R. Form or function? Part 2. Objective cosmetic and functional correlates of quality of life in women treated with breast-conserving surgical procedures and radiotherapy. Cancer, New York, v.15, n.912, p.2282-2287, Jun. 2001.

MATSUOKA, Y.; NAKANO, T.; INAGAKI, M.; SUGAWARA,Y.; AKECHI, T.; IMOTO, S.; MURAKAMI, K.; YAMAWAKI, S.; UCHITOMI, Y. Cancer-related thoughts as an indicator of poor psychological adjustment at 3 or more years after breast surgery: a preliminary study. Breast Cancer Research and Treatment, Dordrecht, v.76, n.2, p.117-124, Nov. 2002.

MCSWEENNY, A. J.; CREER, T. L. Health-Related Qualityof-Life Assessment in Medical Care. Disease-a-Month, Chicago, v.41, n.1, p.1-72, 1995.

MOREIRA, E. C. H.; MANAIA, C. A. R.; GOLIAS, C.; CENTOFANTI, M. D.; SILVA, D. W.; FLORES, D. M. Estudo Comparativo entre o questionário de qualidade de vida específico para pacientes mastectomizadas e o SF-36. Reabilitar; São Paulo, v.21, n.5, p.10-19, 2003.
MOREIRA, E. C. H.; PENASSO, P.; ASSAD, M. A. C.; COUTO FILHO, J. Proposta de elaboração e validação de um questionário de qualidade de vida, específico para paciente submetida ao tratamento cirúrgico de câncer de mama. Reabilitar, São Paulo, v.4, n. 14, p.43-48, 2002.

NAGEL,G. C.; SCHMIDT, S.; STRAUSS, B. M.; KATENKAMP, D. Quality of life in breast cancer patients: a cluster analytic approach: Empirically derived subgroups of the EORTC-QLQ BR 2- a clinically oriented assessment. Breast Cancer Research and Treatment, Dordrecht, v.68, n.1, p.75-87, jul. 2001.

SALES, C. A. C.; PAIVA, L.; SCANDIUZZI, D.; ANJOS, A. C. Y. Qualidade de vida de mulheres tratadas de câncer de mama: funcionamento social. Revista Brasileira de Cancerologia, Rio de Janeiro, v.47, n.3, p.263-272, 2001.

SHIMOZUMA, K.; GANZ, P. A.; PETERSEN, L.; HIRJI, K. Quality of life in the first year after breast cancer surgery: rehabilitation needs and patterns of recovery. Breast Cancer Research and Treatment, Dordrecht, v.56, n.1, p.45-57, jul. 1999.

VELANOVICH, V.; SZYMANSKI, W. Quality of life of breast cancer patients with lymphedema. The America Journal of Surgery, New York, v.177, n.3, p.184-188, 1999.

WHELAN, T. J.; LEVINE, M.; JULIAN, J.; KIRKBRIDE, P.; SKINGLEY, P. The effects of radiation therapy on quality of life of women with breast carcinoma. Cancer, v. 88 , n. 10, p.2260-2266, May, 2000. 
ANEXO 1

QUESTIONÁRIO DE AVALIAÇÃO DA QUALIDADE DE VIDA DA PACIENTE MASTECTOMIZADA

PACIENTE

RG $\mathrm{HU} / \mathrm{HC}$

DATA

ENTREVISTADOR

$\mathrm{n}^{\mathrm{o}}$ da entrevista

Antes de iniciar solicito permissão para tratar a Sra. mais informalmente neste questionário.

Eu gostaria que você pensasse nas atividades das últimas duas semanas que fizeram você sentir limitação, isto é, dificuldade ao realizá-las. Devem ser atividades que você realiza freqüentemente e que são importantes na sua vida diária.

1. Por favor, cite todas as atividades, as quais você tenha feito nas últimas duas semanas que fizeram você sentir dificuldade ao realizá-las. Você pode lembrar de outra atividade que você tenha feito nas últimas duas semanas e que estava limitada?

2. Agora eu vou mostrar uma lista de outras atividades que fazem pessoas que também foram submetidas à mastectomia sentirem dificuldade para realizá-las. Eu vou fazer uma pausa longa o suficiente após cada item, para que você me diga se tem sentido dificuldade durante a realização destas atividades ou se outra pessoa precisou realizá-las por você, durante as últimas duas semanas. Caso esta atividade não precisou ser feita durante as duas últimas semanas responda apenas não. As atividades são:

3. Dos itens que você escolheu quais os mais importantes no seu dia a dia? Eu gostaria que você me mostrasse qual é o mais importante.

Vamos rever se estas foram as atividades limitadas mais importantes nestas últimas duas semanas e vamos colocar em ordem de importância para o seu dia a dia. Atividades de Vida Diária limitadas nas duas últimas semanas

\begin{tabular}{|c|l|c|c|c|c|c|c|c|}
\hline Ordem & Atividades limitadas & 1 & 2 & 3 & 4 & 5 & 6 & 7 \\
\hline 1 & & & & & & & & \\
\hline 2 & & & & & & & & \\
\hline 3 & & & & & & & & \\
\hline 4 & & & & & & & & \\
\hline 5 & & & & & & & & \\
\hline
\end{tabular}

Total de pontos $=$ Soma das notas / 3,5 Obtem-se nota $=$

4. Eu gostaria que você descrevesse o quanto de dificuldade você sentiu nas últimas duas semanas enquanto estava realizando cada umas destas cinco atividades. Olhe este CARTÃO VERDE (EVA)..

Por favor indique só o número correspondente ao quanto limitada, ou seja, o grau de dificuldade de realizar a atividade somente durante as últimas duas semanas . 


\section{LISTA DE ATIVIDADES MOSTRADAS PELO ENTREVISTADOR}

- Pentear o cabelo

- Vestir uma calça

- Vestir uma camiseta (sem botões)

- Vestir uma blusa (com botões)

- Amarrar o sapato

- Almoçar ou jantar

- Segurar um objeto pequeno

- Segurar um objeto grande

- Carregar peso, por exemplo, compras

- Deitar-se

- Lavar a louça

- Atividades profissionais

- Atividades esportivas

- Descascar uma fruta

- Dirigir

- Correr, por exemplo para pegar ônibus

- Levantar-se

- Tomar uma bebida quente, por exemplo café, leite e chá
- prender o cabelo

- tirar camiseta

- pegar um objeto no alto

- estender roupas

- passar esmalte

- depilação

- Escovar os dentes

- Caminhar

- Arrastar a mobília

- Varrer a casa

- Lavar e passar roupas

- Fazer compras no supermercado

- Arrumar a cama

- Preparar suas refeições

- Tomar banho

- Deitar-se

- Brincar com filhos ou netos

- Dançar

Agora vamos fazer 25 perguntas que você responderá baseada neste CARTÃO AZUL (EVA).

Por favor aponte a resposta e lembre-se de ser o mais sincero possível.

QUANTO TEMPO NAS DUAS ÚLTIMAS SEMANAS ...

1 ...vc se sentiu bem em relação a sua aparência física?

2 ...vc notou que seu membro superior do lado da mastectomia estava edemaciado, ou seja, inchado?

3 ....vc se sentiu desejado e/ou atraente?

4...vc se sentiu frustrada, ou seja, triste por não conseguir realizar alguma atividade no seu dia-a-dia?

5 ...vc se sentiu envergonhada em relação à sua aparência física?

6 ...vc sentiu dor no seu membro superior, tórax ou região dorsal ?

7 ...vc se sentiu feliz e satisfeita com sua vida pessoal?

8....vc trocou carícias com seu marido/ namorado/ companheiro?

9 ...vc evitou situações em que sua aparência física poderia ser reparada por outros? (ex: evitou situações sociais, piscinas e contato íntimo)

$10 \ldots \mathrm{vc}$ notou alterações na sua postura? (ex: um ombro mais elevado do que o outro, ombros protrusos, cabeça e mãos anteriorizadas...)

11 ...vc se sentiu deprimida ou perturbada por pensamentos ruins?

$12 \ldots$ ve se sentiu atraída por seu marido/ namorado/ companheiro?

13 ...vc usou roupas largas para esconder a ausência da mama?

14 ...vc sentiu fraqueza muscular no membro superior do lado da mastectomia?

$15 . . . v c$ se sentiu desencorajada e com medo em relação ao futuro?

16 ...vc acreditou que os movimentos do seu membro superior pudessem ficar iguais a antes da mastectomia?

$17 \ldots$ vc procurou arrumar-se, por exemplo, pentear o cabelo, maquiar-se, usar roupas bonitas?

$18 \ldots$ vc evitou se olhar no espelho de corpo inteiro? 
$19 . . . v c$ se sentiu a mesma mulher de antes da cirurgia?

$20 . . . v c$ se sentiu com energia, motivação, disposição?

21 ...vc não pôde trabalhar tão bem quanto antes da mastectomia?

22 ...vc se sentiu apoiada por seus familiares e amigos?

$23 . . . v \mathrm{vc}$ evitou tocar ou olhar diretamente na cicatriz cirúrgica?

$24 \ldots \mathrm{vc}$ pensou em desistir do tratamento medicamentoso ou fisioterapêutico?

25 ...vc se sentiu amada pelo seu marido/ namorado/ companheiro

Nota:

Aparência física (AF): soma-se as notas referentes às perguntas $1^{*}, 5,9,13,18,23$ e divide por 4,2. Assim irá obter a nota máxima 10. As notas das perguntas marcadas com asterisco devem ser invertidas, ou seja, se a paciente aponta a resposta de nota 1 no cartão azul, esta valerá 7 ou vice-versa. Então 1-7, 2-6, 35 e 4 permanece.

Emocional (EM): soma-se as notas referentes às perguntas 4, 7*, 11, 15, 17*, 20*, 22*, 24 e divide por 5,6. Assim irá obter a nota máxima 10. Segue a mesma regra para as perguntas marcadas com asterisco.

Sexualidade (SX): soma-se as notas referentes às perguntas $3^{*}, 8^{*}, 12^{*}, 19^{*}, 25^{*}$ e divide por 3,5. Segue a mesma regra para as perguntas marcadas com asterisco.

Sintomatologia (ST): soma-se as notas referentes às perguntas 2, 6, 10, 14, 16*, 21 e divide por 4,2. Assim obterá a nota máxima 10. Segue a mesma regra para as perguntas marcadas com asterisco.

Média: Média: (LF . 5) + (AF . 6) + (EM . 8) + (SX . 5) + ( ST . 6)/30 